\title{
First documented case of intracranial falcine malignant peripheral nerve sheath tumor: illustrative case
}

\author{
Renato J. Galzio, MD, ${ }^{1}$ Mattia Del Maestro, MD, ${ }^{2}$ Diamantoula Pagkou, MD, ${ }^{3}$ Massimo Caulo, MD, ${ }^{4}$ Sofia Asioli, MD, ${ }^{5}$ \\ Alberto Righi, MD, PhD, ${ }^{6}$ Viscardo Paolo Fabbri, MD, ${ }^{5}$ and Sabino Luzzi, MD, $\mathrm{PhD}^{7,8}$
}

\begin{abstract}
${ }^{1}$ Neurosurgery Unit, Maria Cecilia Hospital, Cotignola, Italy; ${ }^{2} \mathrm{PhD}$ School in Experimental Medicine, and ${ }^{7}$ Neurosurgery Unit, Department of Clinical-Surgical, Diagnostic and Pediatric Sciences, University of Pavia, Pavia, Italy; ${ }^{3}$ Department of Neurosurgery, Aristotle University of Thessaloniki, AHEPA University Hospital, Thessaloniki, Greece; ${ }^{4}$ Department of Neuroscience and Clinical Sciences, University of Chieti, Italy; ${ }^{5}$ Department of Biomedical and Neuromotor Sciences (DIBINEM)-Surgical Pathology Section, University of Bologna, Italy; ${ }^{6}$ Department of Pathology, IRCCS Istituto Ortopedico Rizzoli, Bologna, Italy; and ${ }^{8}$ Neurosurgery Unit, Department of Surgical Sciences, Fondazione IRCCS Policlinico San Matteo, Pavia, Italy
\end{abstract}

BACKGROUND The authors reported the first documented case of intracranial extraaxial nonneurofibromatosis type 1-related nontriton malignant peripheral nerve sheath tumor (MPNST) originating from the falx cerebri.

OBSERVATIONS A 34-year-old man with headache, short-term memory deficit, postural instability, and blurred vision presented with a large heterogenous contrast-enhanced intraventricular cystic lesion originating from the free margin of the falx cerebri. The patient received surgery using the right posterior interhemispheric approach. Gross total resection was performed, and the inferior border of the falx cerebri was resected. The postoperative course was uneventful. Histological examination revealed hypercellular foci of neoplastic spindle cells with hyperchromatic and wavy nuclei. Hence, a diagnosis of MPNST was made based on concomitant immunochemistry findings, including mouse double minute 2 homolog focal positivity and geographic loss of H3K27me3. The patient received adjuvant radiotherapy, and recurrence was not observed.

LESSONS Intracranial MPNSTs are extremely rare tumors, typically originating from the cranial nerves in the posterior cranial fossa. An even rarer variant of these tumors, referred to as malignant intracerebral nerve sheath tumors, may directly arise from the brain parenchyma. The authors reported the first case of an intracranial MPNST originating from the dura mater of the falx cerebri, acting as an extraaxial lesion with prevalent expansion in the right ventricle.

https://thejns.org/doi/abs/10.3171/CASE21255

KEYWORDS H3K27me3; intracranial malignant peripheral nerve sheath tumor; malignant intracerebral nerve sheath tumor; mesenchymal brain tumor

Malignant peripheral nerve sheath tumor (MPNST) is the sixth most common soft tissue sarcoma, with an incidence of 0.1 to 0.2 per 100,000 people annually. ${ }^{1}$ Moreover, it originates from the Schwann cell lineage. Approximately half of MPNSTs are associated with neurofibromatosis type 1 , and the other half occurs spontaneously or after exposure to ionizing radiation. ${ }^{2}$ MPNSTs typically originate from the nerves of the extremities and trunk or from a preexisting benign nerve sheath tumor. ${ }^{3,4}$ Tumors in the head and neck region only account for
$0.4 \%$ to $0.8 \%$ of all MPNST cases. ${ }^{5}$ These malignant lesions have a poor prognosis and a high risk of regional or systemic metastases. Moreover, tumors measuring $>5 \mathrm{~cm}$, those located at the trunk, and those with a high-grade morphology are considered prognostic factors for adverse outcomes. ${ }^{3,6}$ All age groups, from infancy to adulthood, can be affected. Intracranial cases are rare and often classified in two groups based on their relationships with the neurovascular structures primarily involved: (1) extraaxial MPNSTs originating from the cranial

ABBREVIATIONS CBV = cerebral blood volume; $\mathrm{CT}=$ computed tomography; MDM2 = mouse double minute 2 homolog; MINST = malignant intracerebral nerve sheath tumor; MPNST = malignant peripheral nerve sheath tumor; MRI = magnetic resonance imaging.

INCLUDE WHEN CITING Published August 9, 2021; DOI: 10.3171/CASE21255.

SUBMITTED April 25, 2021. ACCEPTED June 24, 2021.

(C) 2021 The authors, CC BY-NC-ND 4.0 (http://creativecommons.org/licenses/by-nc-nd/4.0/). 

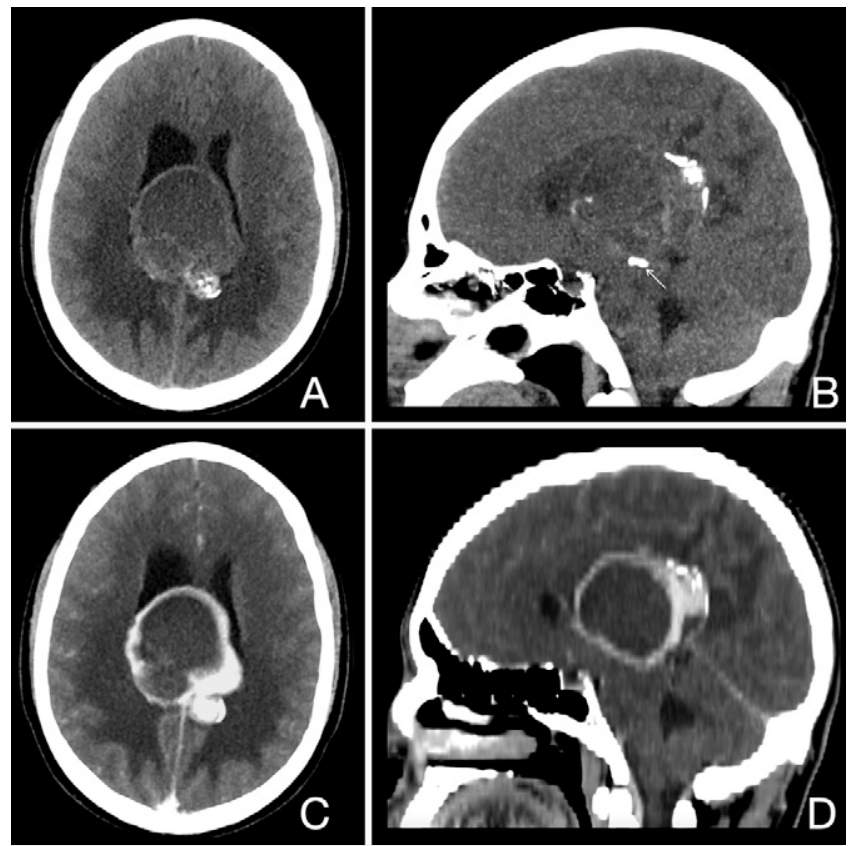

FIG. 1. Axial (A) and sagittal (B) CT scan showing a right ventricular mass and diffuse vasogenic edema. The iodine-based contrast revealed an intensely enhanced tumor wall, with nodular areas of thickening in the left posterolateral portion (C). The left paramedian calcific nodule in the sagittal plane appeared in relation to the inferior border of the falx cerebri (D). nerves in the posterior cranial fossa, more frequently from the vestibular, trigeminal, and lower cranial nerves, and (2) intraparenchymal type, referred to as malignant intracerebral nerve sheath tumor (MINST). ${ }^{7-12}$ The histological, immunohistochemical, and ultrastructural characteristics of MINST are similar to those of MPNST. However, the former originates directly from brain parenchymal tissue, and it has no relationship with the cranial nerves. ${ }^{13-15}$ Pathogenetically, MINSTs are supposed to originate from ectopic multipotential mesenchymal cells of the neural crest, which are displaced during embryogenesis. ${ }^{16}$ Nevertheless, only a few cases have been reported in the literature, and lesions are commonly located in the cerebral hemispheres. ${ }^{4,17-19}$ Herein, we report the first case of a subtype of primary intracranial MPNST originating from the falx cerebri with extraaxial development and ventricular involvement.

\section{Illustrative Case}

A 34-year-old man presented with a 2-month history of persistent headache, short-term memory deficit, slight postural instability, and blurred vision. His previous medical history was irrelevant, and there were no signs of neurofibromatosis. The patient had normal cranial nerve function, motor strength, sensation, and reflexes. However, the cerebellar test revealed slight gait unsteadiness, and short-term memory deficit was noted. Fundoscopy showed bilateral papilledema (Frisen grade II). Noncontrast-enhanced computed tomography (CT) revealed a huge, ovoidal mass embedded in the lateral ventricles, with a thin hyperdense wall containing sparse and small calcifications and slightly hypodense contents. A paramedian nodule with coarse calcifications was observed in the posterior superior paramedian left surface of the lesion, which compressed
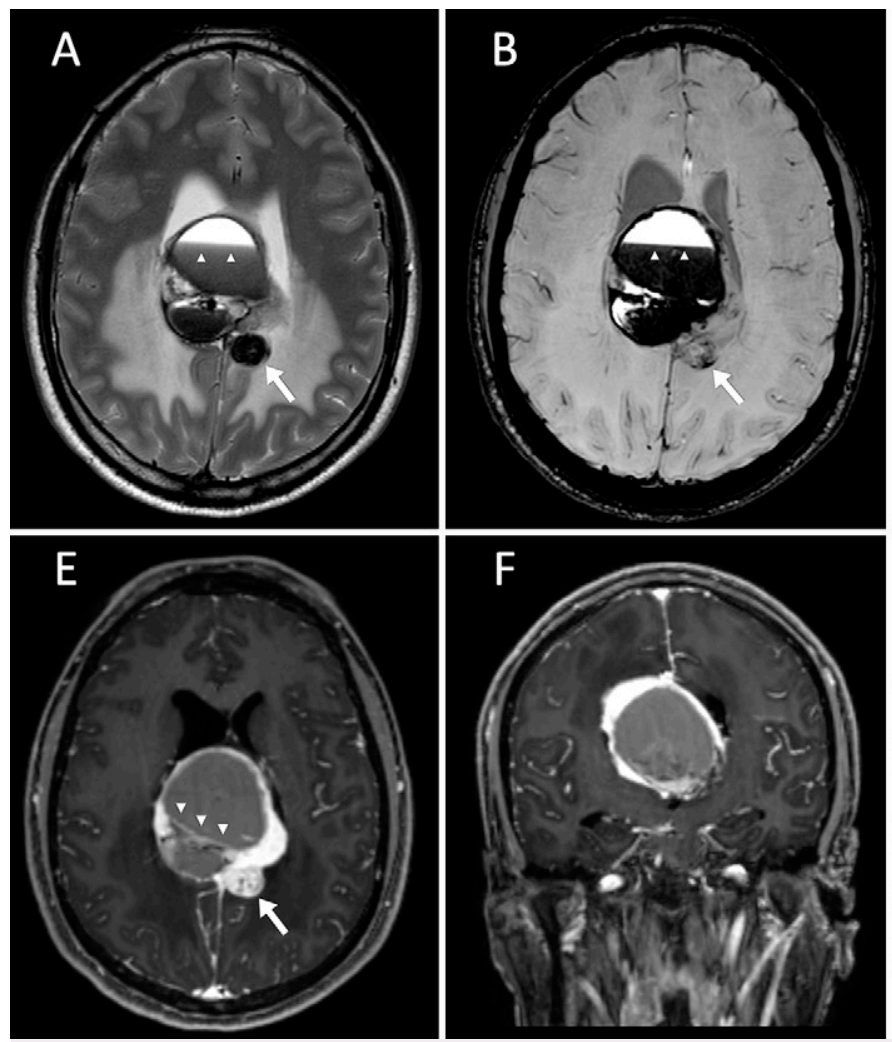
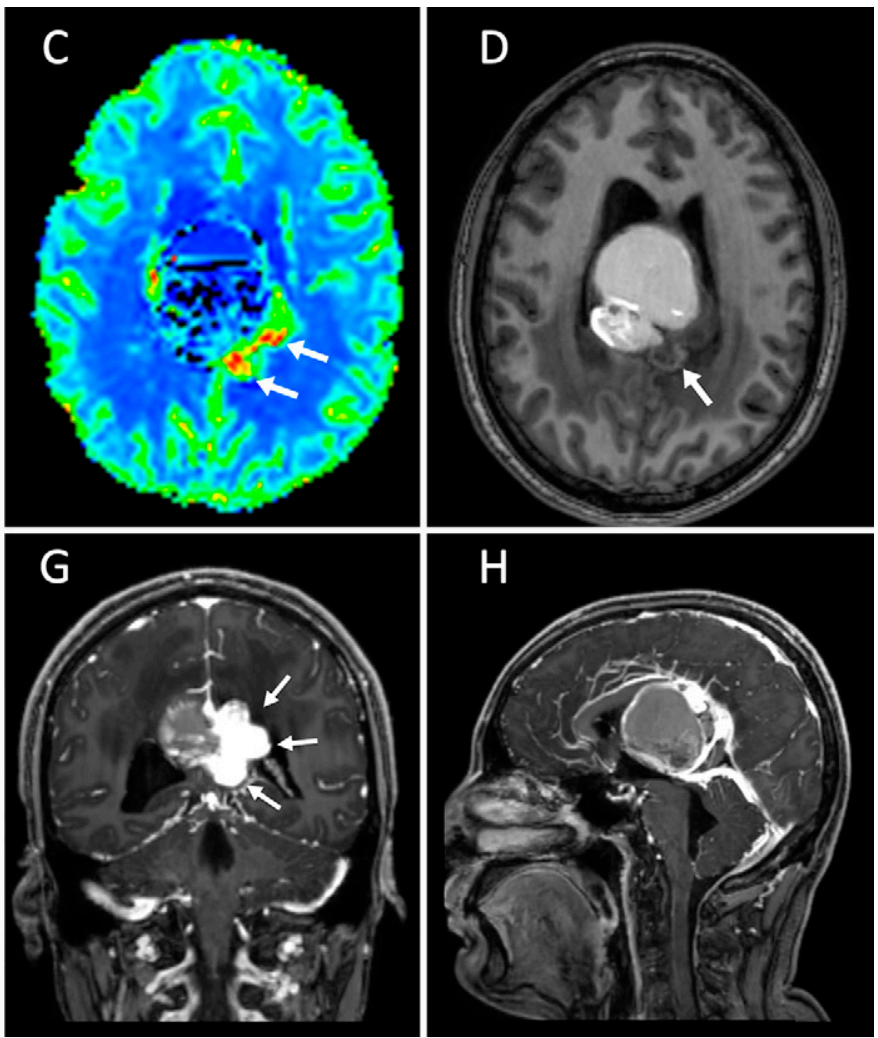

FIG. 2. Axial T1-weighted (A), fluid-attenuated inversion recovery (B), diffusion-weighted (C), and perfusion-weighted (D) MRI. Axial (E), coronal (F and

G), and sagittal (H) contrast-enhanced MRI. Arrows indicate the solid part of the lesion, whereas arrowheads show the inner blood-fluid level. 
the aqueduct, thereby causing incipient supratentorial hydrocephalus. The pineal gland was partially calcified and was detected below and separated from the tumor. Diffuse edema surrounding the lesion was evident (Fig. 1A and B).

Contrast-enhanced CT was subsequently performed, and results showed an intensely enhanced tumor wall, with nodular areas of thickening in the left posterolateral area. In particular, the left paramedian calcified nodules were also intensely enhanced and were in strict contact with the falx cerebri, with evidence of dura thickening at the contact level (Fig. 1C and D). Magnetic resonance imaging (MRI) was performed using a 3T scanner equipped with a 32-channel head coil. The patient received plain and contrast-enhanced standard MR sequences, dynamic susceptibility contrast-enhanced perfusion MRI, and multivoxel MR spectroscopy. A large, almost ovoidal (maximal diameters: sagittal, $59 \mathrm{~mm}$; axial, $52 \mathrm{~mm}$; and coronal, $45 \mathrm{~mm}$ ) significantly heterogenous space-occupying lesion was observed in the right/left paramedian area posterior to the fornix just above the pineal region. The tumor developed in the right lateral ventricle, contralaterally shifting the septum pellucidum, bulging superiorly at the posterior third of the corpus callosum, which appeared stretched and displaced. Bulging was evident in the right side. The third ventricle was compressed and displaced downward and anteriorly. The lesion was characterized by an inner blood-fluid level and smooth and regular walls, except for three posterior, confluent, and superficial solid nodules (Fig. 2A-C). Perfusion MRI showed high cerebral blood volume (CBV) values (five times higher than those of the normal white matter of the frontal lobes, indicative of neoangiogenesis) in the nodular portion (Fig. 2D). MR spectroscopy revealed a slight Cho increase, with a Cho: $\mathrm{N}$-acetylaspartate ratio of 1.7 (indicative of moderate hypercellularity). Contrast- enhanced three-dimensional T1-weighted sequences revealed that the lesion had dura attachment to the inferior margin of the falx cerebri with extraaxial development of the nodular portion toward the mesial surface of the left parietal lobe (Fig. 2E-H). Imaging was completed with contrast-enhanced MRI of the whole spine, and the examination did not reveal any lesion.

With the patient in prone Concorde position, a biparietal craniotomy was performed, exposing the middle third of the superior sagittal sinus. The dura was opened bilaterally and cerebrospinal fluid was obtained through a ventricular catheter inserted in the right occipital horn. Under microscopic view, a right interhemispheric approach enabled identification of the superior surface of the tumor bulging from the split corpus callosum. The lesion had a tense-elastic consistency and appeared reddish, essentially vascularized by several arteries coming from the falx cerebri (Fig. 3A). The tumor cyst was punctured, and its contents, consisting of a collection of blood indicative of chronic hematoma, were evacuated (Fig. 3B). The inferior margin of the falx cerebri was coagulated and sectioned, and the tumor was debulked using the ultrasonic aspirator, shrunk via bipolar coagulation, and resected piece by piece to achieve a gross total resection (Fig. $3 \mathrm{C}$ and F).

In terms of histology, the nodular fragment attached to the dura showed hypocellular and hypercellular foci of neoplastic spindle cells with hyperchromatic and wavy nuclei (Fig. 4A and B). Epithelioid morphology (Fig. 4C) as well as myxoid-rich nodules and perivascular arrangement (Fig. 4D) were noted. Atypical mitotic figures were focally present (5/10 high-power field). Other fragments from the wall of the intraventricular portion of the tumor revealed extensive areas of coagulative necrosis, hemorrhage, fibrosis (Fig. 4E), and sparse neoplastic nodules. Immunohistochemistry showed
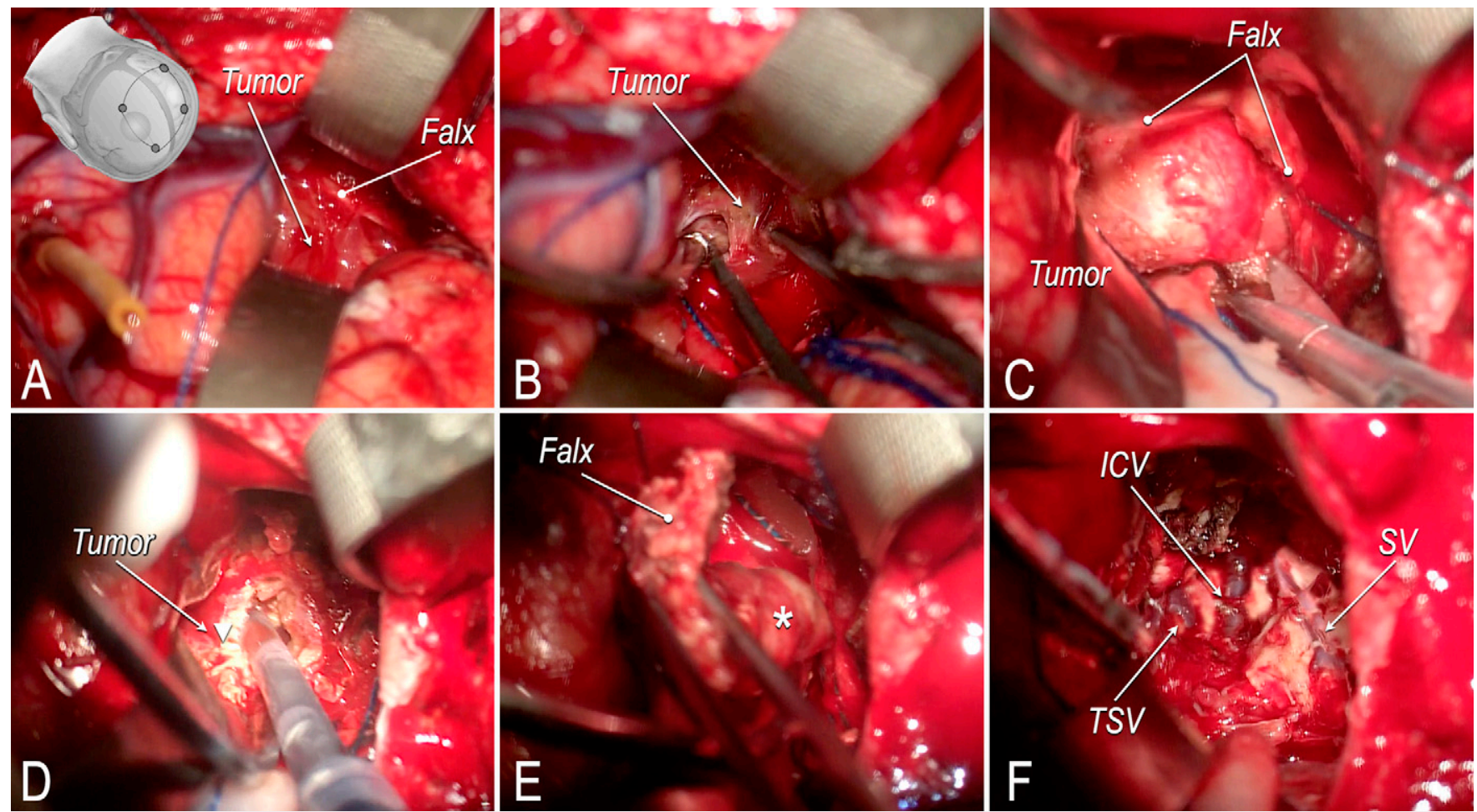

FIG. 3. A-F: Intraoperative images showing the exposure, debulking, and resection of the tumor. ICV, right internal cerebral vein; SV, right septal vein; TSV, right thalamostriate vein. 


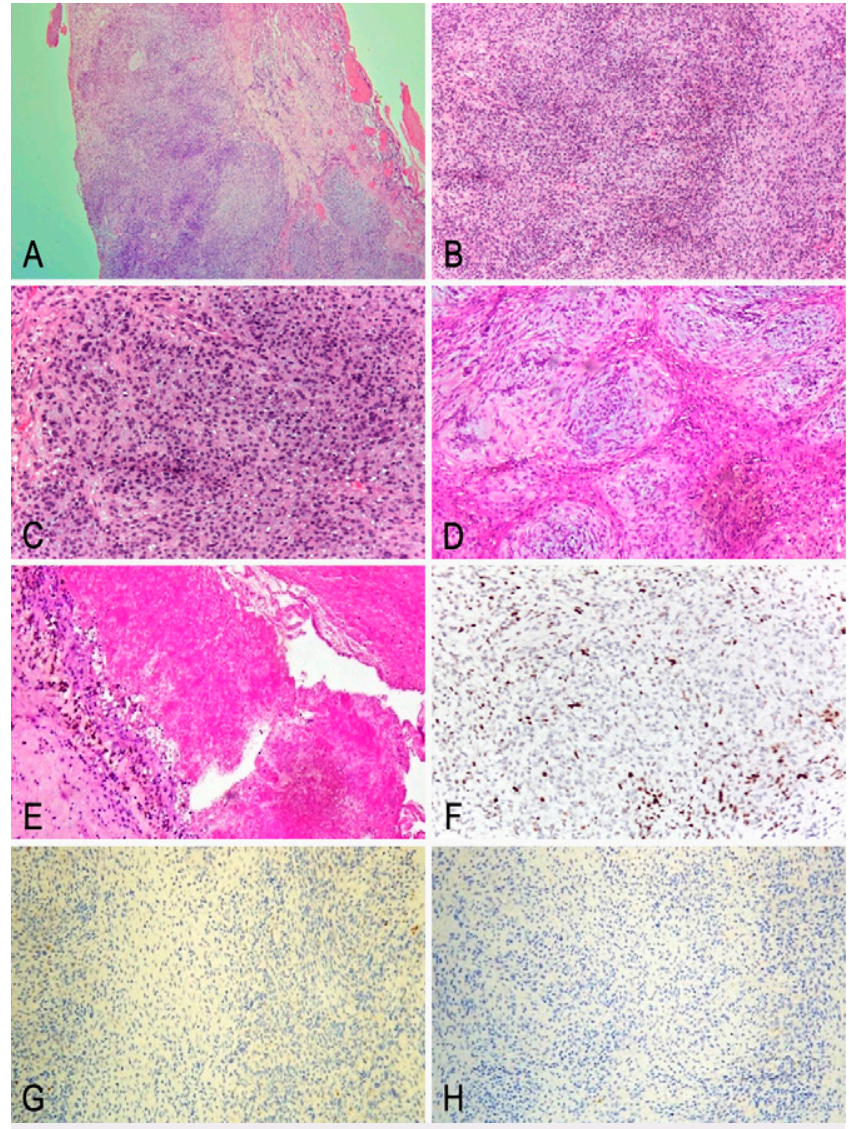

FIG. 4. Histological examination. A: Hematoxylin and eosin staining showing infiltration of the pachymeninges (original magnification $\times 20$ ). B: Hematoxylin and eosin staining (original magnification $\times 100$ ). The tumor comprised spindle and epithelioid proliferation showing transition from a hypercellular to hypocellular area. C: Hematoxylin and eosin staining (original magnification $\times 200$ ). There was clustering of epithelioid neoplastic cells around the blood vessels. D and E: Hematoxylin and eosin staining (original magnification $\times 100$ ). Myxoid-rich nodules were focally observed along with extensive areas of coagulative necrosis, hemorrhage, and fibrosis. Immunohistochemical analysis: (F) shows partial loss of $\mathrm{H} 3 \mathrm{~K} 27 \mathrm{me} 3$ expression (original magnification $\times 200$ ). Negativity for SS18-SSX fusion-specific antibody (G) and SSXspecific antibody $(\mathbf{H})$ designed to the SSX C-terminus.

neoplastic cells with a strong positivity for vimentin and a patchy reaction for epithelial membrane antigens. However, the patient tested negative for glial fibrillary acidic protein, Olig2, progesterone receptor, CD31, erythroblast transformation-specific-related gene, cytokeratin, actin smooth muscle, desmin, S-100, and SOX10. Mouse double minute 2 homolog (MDM2) focal positivity and geographic loss of H3K27me3 were observed (Fig. 4F). To rule out the diagnosis of synovial sarcoma, reverse transcription polymerase chain reaction analysis was performed and did not show SYT-SSX1 and SYT-SSX2 gene rearrangements. These data were confirmed by fluorescence in situ hybridization analysis and immunohistochemistry using SS18SSX fusion-specific antibody and SSX-specific antibody designed to the SSX C-terminus (Fig. $4 \mathrm{G}$ and $\mathrm{H}$ ). ${ }^{20}$ Therefore, the patient was diagnosed with MPNST. The patient was then discharged on postoperative day 5 . Postoperative MRI at 30 days after surgery showed gross total resection of the tumor volume (Fig. 5). The patient received adjuvant radiotherapy at a dose of $60 \mathrm{~Gy}$ in a conventional regimen of 2.0 Gy per fraction. The target volume included the surgical cavity. At 1month follow-up, memory deficit completely resolved. The patient received clinical and radiological MRI quarterly, and there was no evidence of recurrence 13 months after surgery.

\section{Discussion \\ Observations}

The term MPSNT was coined by the World Health Organization. It was used to describe different types of soft tissue sarcoma of ectomesenchymal origin, arising from the peripheral nerve or showing nerve sheath differentiation. However, tumors originating from the perineurium or the peripheral nerve vasculature were excluded. ${ }^{21}$ Intracranial MPNSTs are extraaxial neoplasms affecting the cranial nerves and are associated with the nerve root, particularly affecting the vestibular, trigeminal, and lower cranial nerves. ${ }^{7,9-12,22}$ L'heureuxLebeau and Saliba published a systematic review based on 60 cases of intracranial MPNSTs. ${ }^{23}$

A subtype of intraparenchymal MPNST, which is rarer, was described and referred to as MINST. These tumors are typically found in the cerebral hemispheres, particularly in the frontal, temporal, and parietal lobes ${ }^{4,17}$ and, less commonly, in the cerebellum, $^{24-26}$ lateral ventricles, ${ }^{27,28}$ and brainstem. ${ }^{29}$ The current case is histologically an MPNST with no relationship with any cranial nerves and no intraparenchymal development. Moreover, it originated from the falx cerebri and developed as an extraaxial mass in the ventricular system. To date, only 27 cases of MINST have been described, 25 by Le Fevre et al. in $2016,{ }^{17} 1$ by Mrowczynski et al. in $2018,{ }^{30}$ and 1 by Bruscella et al. in $2020 .^{8}$ The aforementioned series did not include cases of MPNST or MINST originating from the dura mater. In 2007, Celikoğlu et al. reviewed six cases of dura-based intracranial schwannoma of the falx cerebri, with no histological features of malignancy. ${ }^{16}$

Regarding ventricular involvement, one case of malignant schwannoma and another case of malignant triton tumor were described. ${ }^{27,28}$ In the current case, preoperative MRI revealed a heterogenous tumor, with an inner blood-fluid level and smooth and regular walls, except for three solid nodules characterized by intense contrast enhancement with dura attachment to the falx cerebri, extraaxially expanded toward the mesial surface of the left parietal lobe. Preoperatively, cystic meningioma was suspected because of a close relationship with the dura, extraaxial development, gadolinium enhancement, and high CBV values. Intraoperatively, the neoplasm actually originated from the posterior third of the falx cerebri, with a posterior nodular portion growing from the left side of the falx cerebri itself and an anterior cystic portion embedded in the right lateral ventricle. The lesion had a welldefined capsule without any intraparenchymal diffusion or relationship with the cranial nerves. It was essentially vascularized by arteries coming from the falx cerebri, and no vascular afferent from the choroid plexus was evident in the cystic intraventricular component. Complete resection could be performed with exeresis of the involved portion of the falx cerebri. The diagnosis of MPNST was made based on histological examination findings, confirmed by immunohistochemistry, which showed a geographic loss of H3K27me3 in neoplastic cells despite negativity for S-100. Pathognomonic features were commonly found in the dural portion of the tumor. Hence, this is the first 

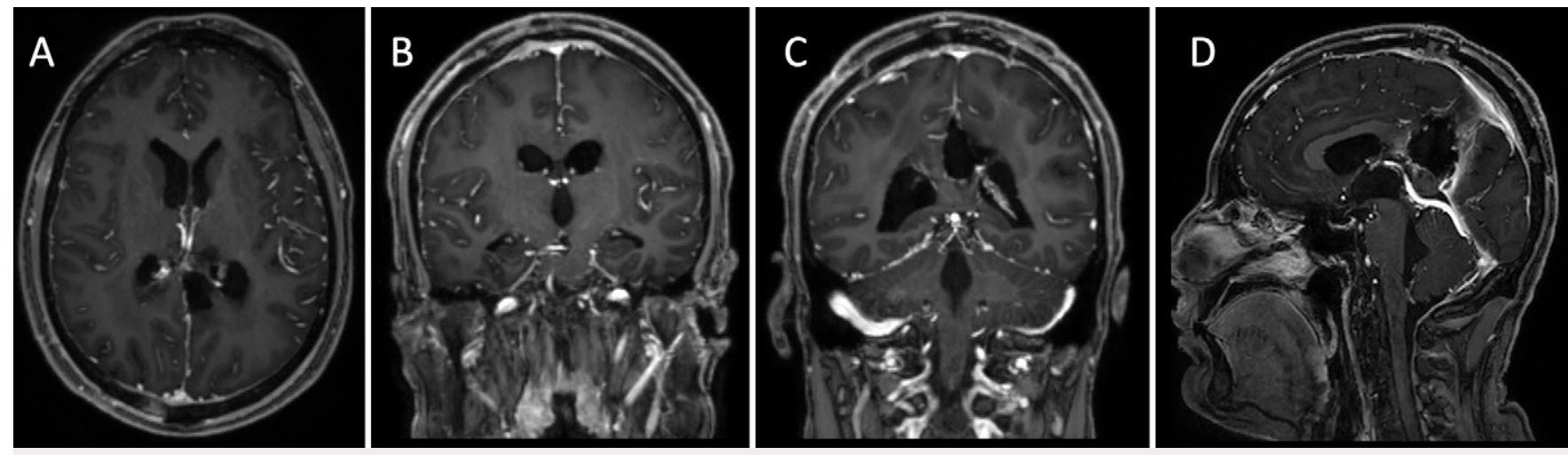

FIG. 5. Axial (A), coronal (B and C), and sagittal (D) contrast-enhanced MRI performed 1 month after surgery.

case of an intracranial MPNST originating from the dura mater and acting as an extraaxial tumor.

In etiopathogenetic terms, typical intracranial MPNSTs arise from the cranial nerves in the posterior cranial fossa, originating from the Schwann cells or from Schwann cells precursors. ${ }^{12,20}$ MINSTs develop in the brain parenchyma without any relationship to intracranial nerves and are considered to originate from multipotential mesenchymal cells of the neural crest displaced during embryogenesis. ${ }^{16,31}$ In the current case, the theory of Horgan et al., which assumes that the tumor originates from small exteroceptive branches of the trigeminal nerve innervating the dura mater in cases of dural attachment, is most acceptable. ${ }^{32}$ This theory is supported by a recent study performed by Kemp et al. That is, the posterior third of the falx cerebri receives innervation from the nervus tentorii of Arnold, a recurrent meningeal branch of the first division of the trigeminal nerve. ${ }^{33}$

The clinical characteristics of MPNSTs and MINSTs are nonspecific and correlated with tumor location and increased intracranial pressure. Intermittent headache, commonly unrelated to head or body position, nausea, vomiting, and visual disturbances are the most common symptoms. Further, papilledema is often observed. Contrast-enhanced MRI is the gold standard in the preoperative workup for these tumors, including the assessment of origin, extension, and involved neurovascular structures. However, no specific pathognomonic features can be used to obtain an accurate differential diagnosis. ${ }^{7,15}$ To date, the diagnosis is solely based on accurate histological examination and immunochemistry findings, including the presence of hypercellular areas comprising pleomorphic epithelioid and spindle cells with atypical mitosis and necrosis arranged in an interlaced fascicular pattern mixed with better-differentiated myxoid nodules. Some studies have shown immunohistochemical positivity for S-100 protein and/or loss of $\mathrm{H} 3 \mathrm{~K} 27 \mathrm{me} 3^{3,34}$ In our case, there was a focal nuclear expression of MDM2, lack of S-100 expression, and loss of H3K27me3.

When managing intracranial MPNST not correlated with the cranial nerves, the differential diagnoses include gliosarcoma, desmoplastic astrocytoma, meningioma, rhabdomyosarcoma, malignant solitary fibrous tumor, and metastasis with spindle cell morphology. ${ }^{4,8}$ Because of the rarity of this tumor, there is no defined treatment protocol, and patients are treated as if they have intracranial mesenchymal sarcoma. Complete resection, which can achieve negative resection margins, is the treatment of choice and has been recommended in previous studies. ${ }^{8,22}$ Total tumor resection, rather than subtotal, is the main prognostic indicator. The aggressive nature of this tumor, with its high rate of local recurrence, indicates that adjuvant radiotherapy is an appropriate treatment strategy. This method increased overall survival within the first 50 months after diagnosis. Therefore, to prevent recurrence, radiation therapy is considered. Because of the high incidence of local recurrence and the tumor's radiosensitivity, local radiation therapy is recommended, particularly after subtotal resection., ${ }^{5,2,22,23,35}$ The dose of adjuvant radiotherapy varies from 50 to 70 Gy (average: $60 \mathrm{~Gy}$ ) based on cerebral localization and the surrounding critical structures. The target volume includes the surgical cavity, which can provide support for volume planning in pre- and postoperative MRI. ${ }^{36,37}$ The effect of adjuvant chemotherapy is still inconclusive. ${ }^{8}$ In the current case, adjuvant radiotherapy at a dose of 60 Gy was delivered in a conventional fractionation regimen. The patient did not present with local recurrence or distant metastasis after more than 1 year.

\section{Lessons}

Clinical characteristics of MPNSTs and MINSTs are nonspecific and correlated with tumor location and increased intracranial pressure. The present case confirms that MPNST may also occur in sites other than cranial nerves of the posterior fossa and brain parenchyma. Accordingly, MPNSTs should be considered in the differential diagnosis of intracranial tumors arising from the falx cerebri. Despite significant advancements in neuroradiological techniques, their diagnosis remains purely histological, and immunocytochemistry plays the most important role. Demonstration of the lack of SS18-SSX fusion in MPNSTs is essential for the differential diagnosis with synovial sarcomas. Maximal safe resection, followed by adjuvant radiation therapy, is strongly recommended to achieve a favorable outcome.

\section{References}

1. Ng VY, Scharschmidt TJ, Mayerson JL, Fisher JL. Incidence and survival in sarcoma in the United States: a focus on musculoskeletal lesions. Anticancer Res. 2013;33(6):2597-2604.

2. Röhrich M, Koelsche C, Schrimpf D, et al. Methylation-based classification of benign and malignant peripheral nerve sheath tumors. Acta Neuropathol. 2016;131(6):877-887.

3. Nielsen GP, Chi P. Malignant peripheral nerve sheath tumour. In: International Agency for Research on Cancer, ed. WHO Classification of Tumours: Soft Tissue and Bone Tumours. IARC Press; 2020:254-257.

4. Shweikeh F, Drazin D, Bannykh SI. Malignant intracerebral nerve sheath tumors: a case report with review of the literature. Case Rep Surg. 2013;2013:384076.

5. Anghileri M, Miceli R, Fiore M, et al. Malignant peripheral nerve sheath tumors: prognostic factors and survival in a series of patients treated at a single institution. Cancer. 2006;107(5):1065-1074. 
6. Banan R, Hartmann C. The new WHO 2016 classification of brain tumors: what neurosurgeons need to know. Acta Neurochir (Wien). 2017;159(3):403-418.

7. Abdel Razek AAK, Gamaleldin OA, Elsebaie NA. Peripheral nerve sheath tumors of head and neck: imaging-based review of World Health Organization classification. J Comput Assist Tomogr. 2020;44(6):928-940.

8. Bruscella S, Alfieri A, de Bellis A, et al. Malignant intracerebral nerve sheath tumor presenting with intratumoral hemorrhage. World Neurosurg. 2021;145:370-375.

9. Guerra-Mora JR, Del Castillo-Calcáneo JD, Córdoba-Mosqueda ME, et al. Malignant nerve sheath tumor involving glossopharyngeal, vagus and spinal nerve with intracranial-extracranial extension and systemic metastases in a patient with type 1 neurofibromatosis: a case report. Int J Surg Case Rep. 2016;29:196-200.

10. Scheithauer BW, Erdogan S, Rodriguez FJ, et al. Malignant peripheral nerve sheath tumors of cranial nerves and intracranial contents: a clinicopathologic study of 17 cases. Am J Surg Pathol. 2009;33(3):325-338.

11. Voorhies J, Hattab EM, Cohen-Gadol AA. Malignant peripheral nerve sheath tumor of the abducens nerve and a review of the literature. World Neurosurg. 2013;80(5):654.e1-654.e8.

12. Ziadi A, Saliba I. Malignant peripheral nerve sheath tumor of intracranial nerve: a case series review. Auris Nasus Larynx. 2010;37(5):539-545.

13. Arumugam G, Ram S, Naidu PB, Kumaravelu S. Pure intracranial, intraparenchymal presentation of a malignant peripheral nerve sheath tumour: a rare case. Neurol India. 2019;67(3):900-903.

14. Barnard ZR, Agarwalla PK, Jeyaretna DS, et al. Sporadic primary malignant intracerebral nerve sheath tumors: case report and literature review. J Neurooncol. 2011;104(2):605-610.

15. Kim HY, Hwang JY, Kim HJ, et al. CT, MRI, and ${ }^{18} \mathrm{~F}-\mathrm{FDG}$ PET/CT findings of malignant peripheral nerve sheath tumor of the head and neck. Acta Radiol. 2017;58(10):1222-1230.

16. Celikoğlu E, Hakan T, Bozbuğa M. Cystic schwannoma of the falx cerebri. J Clin Neurosci. 2007;14(6):589-592.

17. Le Fevre C, Castelli J, Perrin C, Henaux PL, Noel G. Malignant intracerebral nerve sheath tumours: Two case reports and complete review of the literature cases. Article in French. Cancer Radiother. Apr 2016;20(2):119-132.

18. Patankar AP, Sheth JH. Intracranial malignant nerve sheath tumor in the middle cranial fossa: a rare case report with review of literature. Asian J Neurosurg. 2019;14(3):922-926.

19. Son SM, Park YS, Lee OJ. Epithelioid malignant intracerebral nerve sheath tumor: a case report and a comparison with conventional type. J Biomed Transl Res. 2016;17(2):40-45.

20. Baranov E, McBride MJ, Bellizzi AM, et al. A novel SS18-SSX fusion-specific antibody for the diagnosis of synovial sarcoma. Am J Surg Pathol. 2020;44(7):922-933.

21. Woodruff JMKH, Louis DN, Scheithauer BW. Malignant peripheral nerve sheath tumour (MPNST). In: Kleihues PCW, ed. World Health Organization Classification of Tumours: Pathology and Genetics of Tumours of the Nervous System. IARC Press; 2000:172-174.

22. Chen L, Mao Y, Chen H, Zhou LF. Diagnosis and management of intracranial malignant peripheral nerve sheath tumors. Neurosurgery. 2008;62(4):825-832.

23. L'heureux-Lebeau B, Saliba I. Updates on the diagnosis and treatment of intracranial nerve malignant peripheral nerve sheath tumors. OncoTargets Ther. 2013;6:459-470.

24. Beauchesne P, Mosnier JF, Schmitt T, Brunon J. Malignant nerve sheath tumor of the right cerebral peduncle: case report. Neurosurgery. 2004;54(2):500-504.

25. Maiuri F, Colella G, D'Acunzi G, del Bosso De Caro M. Malignant intracerebellar schwannoma. J Neurooncol. 2004;66(1-2):191-195.
26. Singh RV, Suys S, Campbell DA, Broome JC. Malignant schwannoma of the cerebellum: case report. Surg Neurol. 1993;39(2):128-132.

27. Jung JM, Shin HJ, Chi JG, Park IS, Kim ES, Han JW. Malignant intraventricular schwannoma. Case report. J Neurosurg. 1995;82(1):121-124.

28. Takahashi $Y$, Sugita $Y$, Abe T, Yuge T, Tokutomi T, Shigemori M. Intraventricular malignant triton tumour. Acta Neurochir (Wien). 2000;142(4):473-477.

29. Kozić D, Nagulić M, Samardzić M, Ostojić J, Rasulić L, CvetkovićDozić D. Intrapontine malignant nerve sheath tumor: MRI and MRS features. Acta Neurol Belg. 2008;108(2):67-71.

30. Mrowczynski OD, Greiner RJ, Kapadia M, Fanburg-Smith JC, lantosca MR, Rizk EB. Intracranial malignant peripheral nerve sheath tumor variant: an unusual neurovascular phenotype sarcoma case invading through the petrous bone. Childs Nerv Syst. 2018;34(8):1605-1608.

31. van den Munckhof $P$, Germans MR, Schouten-van Meeteren AY, Oldenburger $F$, Troost D, Vandertop WP. Recurring intracranial malignant peripheral nerve sheath tumor: case report and systematic review of the literature. Neurosurgery. 2011;68(4):E1152-E1159.

32. Horgan MA, Kernan JC, Delashaw JB, Schwartz MS, Kuether T. Schwannoma of the torcula presenting as an occipital mass. Case illustration. J Neurosurg. 1998;89(3):490.

33. Kemp WJ 3rd, Tubbs RS, Cohen-Gadol AA. The innervation of the cranial dura mater: neurosurgical case correlates and a review of the literature. World Neurosurg. 2012;78(5):505-510.

34. Prieto-Granada CN, Wiesner T, Messina JL, Jungbluth AA, Chi P, Antonescu CR. Loss of H3K27me3 expression is a highly sensitive marker for sporadic and radiation-induced MPNST. Am J Surg Pathol. 2016:40(4):479-489.

35. McConnell YJ, Giacomantonio CA. Malignant triton tumors: complete surgical resection and adjuvant radiotherapy associated with improved survival. J Surg Oncol. 2012;106(1):51-56.

36. Chao KS, Wippold FJ, Ozyigit G, Tran BN, Dempsey JF. Determination and delineation of nodal target volumes for head-and-neck cancer based on patterns of failure in patients receiving definitive and postoperative IMRT. Int J Radiat Oncol Biol Phys. 2002;53(5):1174-1184

37. Wong WW, Hirose T, Scheithauer BW, Schild SE, Gunderson LL. Malignant peripheral nerve sheath tumor: analysis of treatment outcome. Int J Radiat Oncol Biol Phys. 1998;42(2):351-360.

\section{Disclosures}

The authors report no conflict of interest concerning the materials or methods used in this study or the findings specified in this paper.

\section{Author Contributions}

Conception and design: Galzio, Del Maestro, Pagkou, Righi, Fabbri, Luzzi. Acquisition of data: Galzio, Pagkou, Caulo, Asioli, Righi, Fabbri. Analysis and interpretation of data: Del Maestro, Pagkou, Caulo, Asioli, Righi, Fabbri. Drafting the article: Galzio, Del Maestro, Caulo, Asioli, Fabbri. Critically revising the article: Galzio, Righi, Fabbri. Reviewed submitted version of manuscript: Galzio. Administrative/technical/ material support: Luzzi. Study supervision: Galzio, Luzzi.

\section{Correspondence}

Renato J. Galzio: Maria Cecilia Hospital, Cotignola, Italy. renato.galzio@gmail.com. 